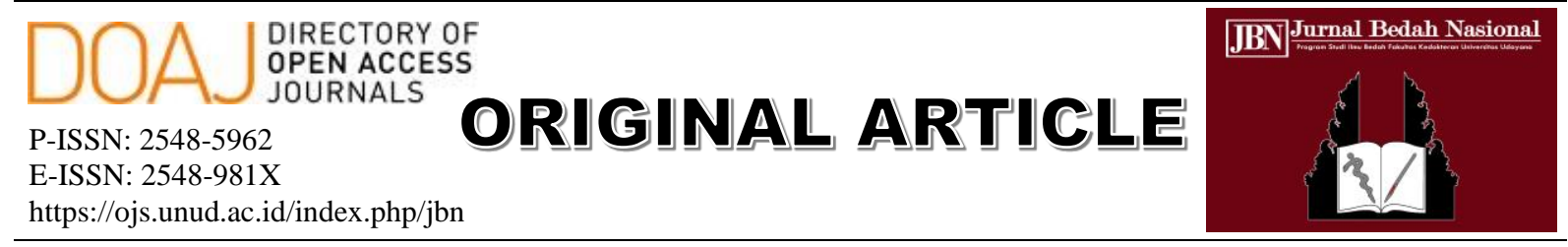

https://ojs.unud.ac.id/index.php/jbn

\title{
Demographic and Clinical Profile of Tuberculous Mastitis Patients at Tebet General Hospital, Jakarta
}

\author{
Desak Ketut Nari Swari Pramegia ${ }^{1}$, Rio Putratama Achmad Faried ${ }^{1}$, Kelvin Setiawan ${ }^{2 *}$, \\ Bramastha Aires Rosadi', Terry Renata Lawanto ${ }^{1}$ \\ ${ }^{1}$ Department of Surgery, Tebet General Hospital, South Jakarta, Indonesia \\ ${ }^{2}$ Department of Surgery, Atma Jaya Faculty of Medicine, North Jakarta, Indonesia \\ *Corresponding author: kelvin.setiawan.a@gmail.com.
}

\begin{abstract}
Aim: Objective of the study is to find out the demographic and clinical profile of tuberculous mastitis (TM) patients. Methods: The study was carried out for a period of one year from August 2017 to August 2018 at Tebet General Hospital. Histopathologically diagnosed cases of TM were retrospectively studied. Results: All eight patients diagnosed as suffering from TM were females with the mean age of 37.25 years. Of these, two patients were lactating, six others were not, and $75 \%$ were multiparous. Unilateral involvement was observed in all cases. The right breast was affected in $62.5 \%$ and left breast in $37.5 \%$ cases. Patients presented with a solitary lump on one breast (87.5\%), swelling of the breast (37.5\%), and breast pain (100\%). In the present study, two of the patients had previous positive tuberculosis (TB) contact and more than half found to be below an average economic level. All the patients were negative on the HIV test; also, there was no focus of TB elsewhere in the body. Conclusion: Tuberculosis should be considered as one possible cause of either mastitis or breast abscess. Histopathology examination played an important role in the diagnosis of TM. Standardized sequence for the diagnosis of TM is needed to increase awareness of the disease.
\end{abstract}

Keywords: mastitis, tuberculous mastitis, breast tuberculosis, granulomatous lesions, extra pulmonal TB.

DOI: https://doi.org/10.24843/JBN.2019.v03.i02.p01

\section{INTRODUCTION}

Tuberculosis (TB) has widely known as a pulmonary disease. In fact, isolated extrapulmonary manifestation constitutes almost $18 \%$ of all TB incidence. ${ }^{1}$ Skin and breast, both count only less than $0.5 \%$ of all cases. $^{2}$ Tuberculous mastitis (TM) is an extremely uncommon condition even in the areas where $\mathrm{TB}$ is endemic with the incidence of 3 to $4.5 \% .^{3} \mathrm{TM}$ was first documented in medical literature in 1829 and since then it has been widely distributed with the most common cases was in India, which might be related to its high rates of tuberculosis. ${ }^{4} \mathrm{TM}$ mostly affects young women in their reproductive age with the most usual presenting symptom is a solitary lesion in one side of the breast. Several factors including parity, history of lactation, trauma, and immunocompromised condition such as AIDS have been reported to be significant risk factors of this condition. ${ }^{3} \mathrm{TM}$ can be either a primary condition in which there is no extra-mammary focus found on the other site, or it can be secondary condition in which pulmonary tuberculosis is positive in the first place. Primary TM is very uncommon. Polymerase chain reaction (PCR) may be a good option for facilitating early diagnosis. However, the gold standard of diagnosis is through culture gained from breast tissue. Unfortunately, acid-fast-bacilli (AFB) detected only in $12 \%$ of the patients. In high burden TB countries, granuloma detected on 
fine needle aspiration means the need for empiric TB therapy even without confirmed acid-fast-bacilli and culture results. In this study, we would like to find out the demographic and clinical profile of tuberculous mastitis (TM) patients.

\section{METHODS}

The study was carried out for a period of one year from August 2017 to August 2018 at Tebet General Hospital. Total eight patients were clinically diagnosed as tuberculosis mastitis, confirmed by biopsy, were included in this study. Data collected through TB DOTS (Directly Observed Treatment, Short Course) center include a detailed history, AFB smear, sputum PCR for Mycobacterium tuberculosis, and HIV test. Histopathologically diagnosed cases of TM were retrospectively studied.

\section{RESULTS}

In present study all eight patients of breast tuberculosis were females, with mean age of 37.25 years old, the youngest one was 23 years old, and the oldest was 52 years old (Figure 1).

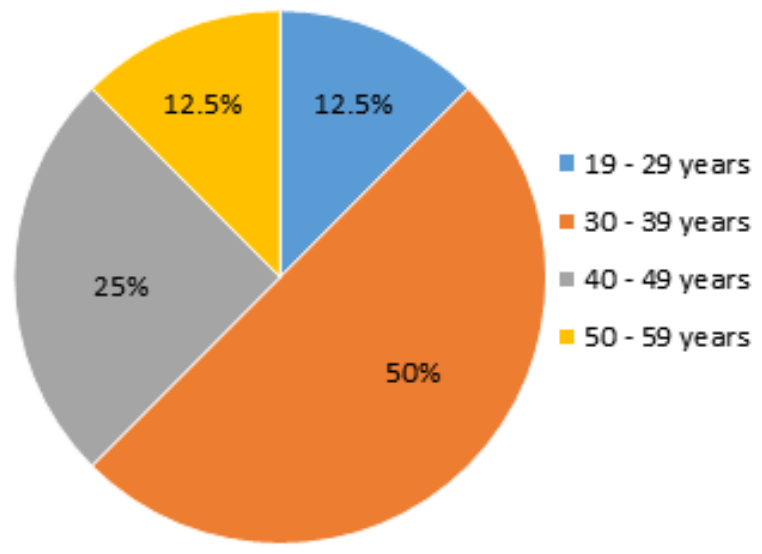

Figure 1. Distribution of tuberculous mastitis by ageranges.

All patient complained of painful breast, seven presents with a solitary lump on their breast, along with breast swelling (Figure 2). In five patients $(62.5 \%)$, right side was involved and in $3(37.5 \%)$ left was involved. The 2 of patients were lactating mothers and one of them was lactating her TB-positive child. Except one who was having only one child, all were multiparous (Table 1).

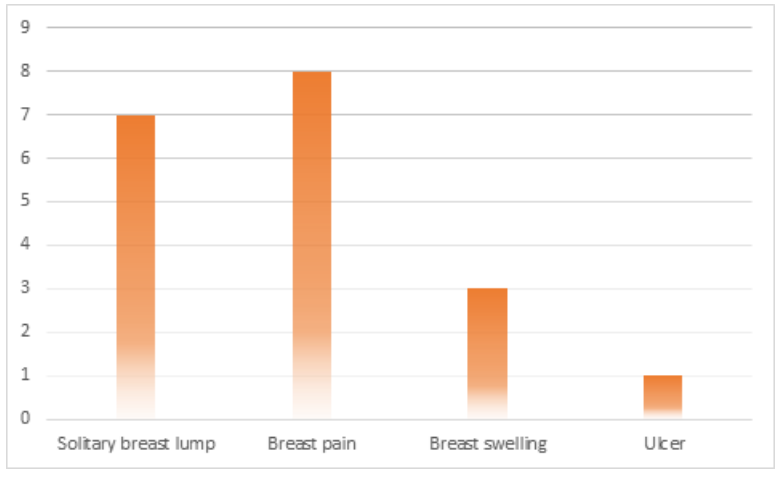

Figure 2. Clinical Symptoms Presented in TM Patient.

The mean duration of presenting symptoms to diagnosis was two months, shortest was two weeks and longest was four months (Table 1). None had respiratory symptoms, but constitutional symptoms such as fever and weight loss were presented in $50 \%$ patients. All patients had primary breast tuberculosis although two had positive previous contact to their family member who were having on-going treatment for TB. This was confirmed by PA lungs radiographs by pulmonary disease specialists that showed no specific signs of pulmonary tuberculosis. There was no family history detected for breast lump or disease. All patients were from rural areas and were housewife's with below average economical level.

Another supporting diagnosis for pulmonary TB including sputum AFB staining and PCR for Mycobacterium tuberculosis were done and negative in all patients (Table 1). Also, none of the patient had positive HIV status or other immunesuppressive disease. Breast biopsy was done and granulomatous lesion along with Langhans giant cell were detected on each patient, consistent with tuberculous mastitis 
(Table 1). Anti-tuberculosis was started in all the cases and gave satisfactory result.

Table 1. Demographic and clinical data of the tuberculous mastitis

\begin{tabular}{|c|c|}
\hline Characteristics & n (\%) \\
\hline \multicolumn{2}{|l|}{ Marital status } \\
\hline Single & 0 \\
\hline Married & $8(100)$ \\
\hline \multicolumn{2}{|l|}{ Education } \\
\hline Elementary school & $1(12.5)$ \\
\hline Junior High school & $2(25)$ \\
\hline Senior High School & $5(62.5)$ \\
\hline \multicolumn{2}{|l|}{ Husband's occupation } \\
\hline Driver & $2(25)$ \\
\hline Employee & $3(37.5)$ \\
\hline Labor & $1(12.5)$ \\
\hline Teacher & $1(12.5)$ \\
\hline Deceased & $1(12.5)$ \\
\hline \multicolumn{2}{|c|}{ Involved part of the breast } \\
\hline Right & $5(62.5)$ \\
\hline Left & $3(37.5)$ \\
\hline \multicolumn{2}{|l|}{ Duration of Symptoms } \\
\hline $0-1$ month & $1(12.5)$ \\
\hline 1-2months & $3(37.5)$ \\
\hline $2-3$ months & $1(12.5)$ \\
\hline 3-4 months & $3(37.5)$ \\
\hline \multicolumn{2}{|l|}{ Lactation } \\
\hline Lactating & $2(25)$ \\
\hline Non-lactating & $6(75)$ \\
\hline \multicolumn{2}{|c|}{ Direct contact to TB Patient } \\
\hline Positive & $2(25)$ \\
\hline Negative & $6(75)$ \\
\hline \multicolumn{2}{|l|}{ Sputum AFB-smear } \\
\hline Positive & 0 \\
\hline Negative & $8(100)$ \\
\hline \multicolumn{2}{|l|}{ HIV Status } \\
\hline Positive & 0 \\
\hline Negative & $8(100)$ \\
\hline
\end{tabular}

\section{DISCUSSION}

Tuberculous mastitis is an atypical disease of the breast with a very low rate of incidence of around 3 to $4.5 \%$ in areas where TB is very common. ${ }^{3}$ It has been said that its rarity is due to breast tissue, as in spleen and skeletal muscle has high protection against tubercle bacilli. ${ }^{5} \mathrm{TM}$ found between the range of 0.025 to $3 \%$ of all patients with surgically treated mammary disease. ${ }^{6}$ Based on the previous study, breast tuberculosis mostly presented in reproductive age due to undergoing frequent hormonal changes during their reproductive life and patients in this range of ages are more prone to trauma and infection. ${ }^{7}$ Mukerjee et al. showed that $76 \%$ of the total subjects of 38 breast tuberculosis patients were in the $21-40$ years old group, while the remaining $24 \%$ were in the 41-70 years old. ${ }^{8}$ In our study, we found the result consistent with the previous study in which $62.5 \%$ of the patients were in the 20-40 years old.

Based on the previous study, 30\% out of 100 patients were lactating at presentation. In other studies, $7 \%$ out of 52 were lactating. While in our study, two patients $(25 \%)$ were lactating. A series from India stated that there might be a correlation between Mycobacterium tuberculosis found in tonsils of breastfed babies with higher rates of mammary tuberculosis in the woman who lactate. In our case, one of TM patient's baby was undergoing TB treatment. Besides parity and history of lactation, HIV infection is also known to be the major risk factor for breast tuberculosis. ${ }^{3}$ However in our study, all the patients had negative HIV infection.

TM is called primary when there is no extra-mammary focus found on the other site. ${ }^{3}$ Primary type of TM of the breast is very rare. Skin abrasions, nipple duct opening, or ribs underneath the affected breast were thought to be the route of infection. Most of TM is usually secondary to another tuberculous focus on the other site of the body. This form can extend via hematogenous, lymphatic, or direct routes and is not always detected through clinical symptoms or radiologic examination. ${ }^{4}$ In the present study, all the patients had primary tuberculous mastitis since there was no other TB focus confirmed elsewhere in the body concluded based on history taking, 
radiological examination, and laboratory studies.

In the previous study, the clinical presentation of TM was usually breast mass, pain, and an open wound with positive discharge. Another study found that breast mass was the highest physical finding in TM patients with the major initial complaint was swelling of the breast. ${ }^{9}$ Similar to the study conducted by Marinopoulos et al., other studies19 also stated that the most common clinical presentation of TM was also a lump, either with or without a duct and sometimes can be painful as well. The lump could somehow mimic the presentation of breast carcinoma in which it was hard, fixed to either the skin or muscle with an irregular border. This condition may be accompanied by inflammation, forming an abscess, causing ulceration and diffuse inflammation of the breast. If there is no adequate response of all these conditions at surgical drainage along with antibiotic treatment, the diagnosis of TM has to be taken into count. ${ }^{5}$

In our study, we found that TM patients came mostly with breast pain, lump, and swelling. Only one of all patients presented with an ulcer. Consistent with the previous study, the clinical presentation of TM still mimics other cause of mastitis or breast lump, so further examination should ideally be done to prevent delay of the diagnosis.

Radiological modalities such as mammography or ultrasonography are described as unreliable in diagnosing breast tuberculosis. ${ }^{10}$ PCR may be offering better accuracy that AFB smear. In our study, each patient underwent AFB smear and sputum PCR. All showed a negative result for Mycobacterium tuberculosis. The most accurate diagnosis of TM is actually through a culture of the breast tissue specimen. However, acid-fast-bacilli detected only in $12 \%$ of the patients. Fine Needle Aspiration and Cytology (FNAC) is helpful in the diagnosis of mammary TB. ${ }^{4,11}$ Khanna et al. showed a $100 \%$ of success in his series while Kakkar et al. found a $73 \%$ of success. ${ }^{9,11}$ In TB-prevalent countries, granuloma confirmed in fine needle aspiration represent the need for empirical TB therapy even when there is no acid-fast-bacilli or positive culture results available. ${ }^{11}$

In our study, we found that the demographic and clinical characteristic of TM itself are often not specific enough to direct clinicians to consider TB as the main cause of the symptoms. This, however, might lead to variable and inconsistent approach or diagnostic sequence of the disease on each patient. Sonography in our study was only done in two patients, obtaining one patient considered as having mastitis, and the other one was having lesion suspicious to carcinoma of the breast. In the end, the definite diagnosis of TM was obtained through surgical biopsy in which all of the histopathological results showed granulomatous lesions with Langhans giant cells, supporting the diagnosis of TM. The seven of eight patients received the standard antitubercular treatment for 9-12 months and has satisfactory result, while one patient was dropped out for halting the treatment in the first five months.

\section{CONCLUSION}

In conclusion, tuberculous mastitis is a very rare type of extrapulmonary tuberculosis which hardly noticed in our daily practice. The diagnosis of TM remains challenging and its symptoms often mimicking other disease. Tuberculosis, especially in rural areas where it is endemic, should be considered as one possible cause of either mastitis or breast abscess. Sonography remains inconclusive, while histopathology examination played an important role in the 
diagnosis of TM. Standardized sequence for the diagnosis of TM is needed to increase awareness of the disease.

\section{ACKNOWLEDGEMENT}

None.

\section{DISCLOSURE}

Researchers declare no conflict of interest of this research.

\section{REFERENCES}

1. Cowie RL, Sharpe JW. Extra-pulmonary tuberculosis: a high frequency in the absence of HIV infection. Int J Tuberc Lung Dis. 1997;1:159-62.

2. Thompson KS, Donzelli J, Jensen J, et al. Breast and cutaneous mycobacteriosis: diagnosis by fine-needle aspiration biopsy. Diagn Cytopathol. 1997;17:45-9.

3. Thimmappa D, Mallikarjuna MN, Vijayakumar A. Breast tuberculosis. Indian J Surg. 2015;77:1378-84.

4. Gupta PP, Gupta KB, Yadav RK, et al Tuberculous mastitis: A review of seven consecutive cases. Indian $J$ Tub. 2003;50:47-50.
5. Marinopoulos S, Lourantou D, Gatzionis T, et al. Breast tuberculosis: Diagnosis, management and treatment. Int J Surg Case Rep. 2012;3:548-50.

6. Singal R, Dalal AK, Dalal U, et al. Primary tuberculosis of the breast presented as multiple discharge sinuses. Indian J Surg. 2013;75:66-7.

7. Kumar M, Chand G, Nag VL, et al. Breast tuberculosis in immunocompetent patients at tertiary care center: A case series. J Res Med Sci. 2012;17:199-202.

8. Mukerjee P, George M, Maheshwari HB, et al. Tuberculosis of the breast. $J$ Indian Med Assoc. 1974;62:410-2.

9. Kakkar S, Kapila K, Singh MK, et al. Tuberculosis of the breast. A cytomorphologic study. Acta Cytol. 2000;44:292-6.

10. Khodabakhshi B, Mehravar F. Breast tuberculosis in Northeast Iran: review of 22 cases. BMC Womens Health. 2014;14:72.

11. Khanna R, Presanna G V, Gupta P, Kumar M, Khanna S, Khanna A K. Mammary tuberculosis: report on 52 cases. Postgrad Med J. 2002;78:422-4. 